\title{
Addressing Fear Avoidance and Self-efficacy Beliefs to Reduce Disability in Patients with Non-specific Low Back Pain: From Theory to Application
}

\section{George A Koumantakis ${ }^{1,2 *}$}

${ }^{1}$ Assistant Professor, Department of Physiotherapy, University of West Attica

(UNIWA), Athens, Greece

${ }^{2}$ Director, Laboratory of Advanced Physiotherapy (LAdPhys), Department of

Physiotherapy, University of West Attica (UNIWA), Athens, Greece

*Corresponding Author: George A Koumantakis, Laboratory of Advanced

Physiotherapy (LAdPhys), Department of Physiotherapy, University of West Attica

(UNIWA), Athens, Greece.
Received: October 27, 2020

Published: November 18, 2020

(C) All rights are reserved by George A

Koumantakis.

\section{Abstract}

A considerable proportion of patients with non-specific low back pain (nsLBP) report continued symptoms for an extended period of time. In many cases no structural lesion can be identified. Biopsychosocial models attempt to identify the contribution of several psychosocial factors contributing to the development and maintenance of chronic disability in these patients. The fear avoidance model postulates that patients may respond to pain with an avoidance behavior, reducing the level of their activities, resulting in a vicious cycle of prolonged disability, disuse and further pain experience.

The aim of this mini-review is to present some of the most relevant evidence on the interrelationship of fear avoidance as well as self-efficacy beliefs with disability in patients with nsLBP and physical therapy-related methods targeting disability improvement through fear amelioration techniques.

It is concluded that fear is a contributing factor leading to greater levels of disability in individuals suffering an acute episode and is also influencing patient disability further in the subacute and chronic stage of symptoms, also affecting LBP recurrence rate. Results of some studies indicate that there may be additional cognitive factors, like self-efficacy, influencing the outcome of therapy, mainly for those experiencing acute back pain symptoms.

Further investigations should take into account and examine the joint contribution of such psychological factors in order to implement effective and targeted therapy interventions for patients with back pain.

Keywords: Cognitive Factors; Psychosocial; Chronicity; Physical Therapy; Graded Exposure; Pain Neuroscience Education

\section{Abbreviations}

CNS: Central Nervous System; nsLBP: Non-specific Low Back Pain; ALBP: Acute Low Back Pain; CLBP: Chronic Low Back Pain; FAM: Fear-Avoidance Model; FABs: Fear-avoidance Beliefs, FABQ: Fearavoidance Beliefs Questionnaire; TSK: Tampa Scale of Kinesiophobia

\section{Introduction}

Non-specific low back pain (nsLBP) appears to be a common and self-limiting condition. However, for a proportion of individuals symptoms recur over time. These patients who do not recover within a few weeks may develop chronic pain [1], transforming this condition in a major medical, social and economic problem [2]. Croft., et al. (1998), after having examined the outcome of episodes of LBP in a large population, concluded that even though $90 \%$ of 
patients ceased to consult in a period of 3 months, a majority of them had substantial LBP and related disability, with only a $25 \%$ of consulting patients having fully recovered after one year [3]. Back pain may restrict social and other activities and may lead to work loss [4]. Maniadakis and Gray (2000) carried out an economic analysis in UK in 1998, concluding that direct economic burden was around 1.6 billion dollars, while the cost of informal care and the subsequent production losses was approximately 6.5 times the direct cost [5]. Also, in other countries more recent studies have found the cost attributed to LBP excessive, although this varied between countries [6]. Andronis., et al. (2016) on the other hand promoted the cost-effectiveness of exercise therapies for the management of LBP [7].

For decades biomechanical models either based on spinal loading and degeneration [8] as well as abnormal muscle activity in patients [9] had a predominant role in the explanation of back pain. Mechanical stresses to the spine during activities, such as prolonged positions and lifting, were thought to be related with injury of the spinal tissues and having a direct influence on pain and disability [10]. As a consequence, bed rest and avoidance of activities were among the most usual treatment strategies for LBP patients, to promote healing of mechanically stressed tissues [11]. However, it progressively became apparent that the biomedical model could not adequately explain pain and disability levels in ns-LBP, as there was no clear relationship between the amount of tissue damage and the severity of pain experienced by these patients [12].

Therefore, many models have arisen since, incorporating psychological and behavioral factors which in fact offered a better explanation of pain experience and persistent disability [13], with the Fear Avoidance Model (FAM) recognizing the moderating role of fear-avoidance in the treatment outcome of nsLBP patients $[13,14]$. Key cognitive variables in this model are pain catastrophising and pain-related fear, which manifest as a specific fear of movement or re-injury [15]. Some patients are fearful that some typical movements or activities which involve the spine, for example twisting, bending, will cause them serious damage. Two main different behaviors that patients follow when in pain are postulated, these of confrontation and avoidance [16-18]. It seems that the majority of patients in the absence of a serious physical pathology confront daily activities, despite pain. This positive adaptive response might lead to reduction of fears, thus facilitating recovery. Conversely, avoiders anticipate that daily activities might increase pain. As a consequence, they start avoiding these certain activities or movements. When this behavior persists over time, detrimen- tal effects can be occur, such as increased disability, disuse and a lower pain threshold. A vicious circle then may start, with greater fear, inactivity, disability and higher levels of pain [19,20]. The term 'neurosignature' has been proposed, to describe a number of neurons in the central nervous system (CNS) being activated towards a certain task, based on the neuromatrix theory of pain $[21,22]$. In the presence of cognitive influences, such as pain-related fear, the neurosignatures are altered, denoting abnormally increased activity for a certain task $[23,24]$. A number of studies have been conducted concerning fear avoidance and its consequences in nsLBP patients, in order to validate the FAM [25]. Propositions to enhance the theoretical framework of the FAM suggest adoption of a more motivational perspective of that model and to focus on the dynamics of functional recovery, rather than overemphasizing the role of phobias, anxiety and catastrophising [14,26].

\section{Aim of the Study}

The aim of this review is to further address the contributions of a key variable of the FAM, this of fear avoidance beliefs (FABs), in disability and poor behavioral performance of LBP patients and critically discuss newer information contributing further to the validity of this model in LBP patients, even from the acute stage of symptoms. Also, to present treatment interventions targeting fear extinction, while in parallel applying physical therapy interventions promoting physical function in nsLBP, under psychologicallybased methods of delivery.

\section{Methods}

A systematic search strategy was developed in the English language. Online search was performed in the following databases: PubMed, The Cochrane Library, Medline, Google Scolar and PEDro. Reference lists of all identified papers were searched for additional potentially relevant publications. The following search terms were used: LBP, psychosocial factors, disability, fear avoidance beliefs, self-efficacy, physical therapy.

The articles initially identified were numerous $(n=816)$. After exclusion of duplicate publications, reading the abstracts of all primarily identified articles, only select studies that were considered as the most relevant to the contribution of fear avoidance beliefs to disability were included.

\section{Contribution of fear avoidance beliefs (FABs) in LBP disability}

This section presents relevant studies concerning the development and contribution of FABs in acute and chronic LBP patients. 
Addressing Fear Avoidance and Self-efficacy Beliefs to Reduce Disability in Patients with Non-specific Low Back Pain: From Theory to Application

A critical discussion of the main limitations and results of these investigations and the presence of other psychological factors that may contribute to disability in this patient population sums up the salient points of these studies. Moreover, the role of targeted physical therapy interventions for patients that are more prone to develop FABs is emphasized.

\section{FABs in chronic LBP}

Initial studies have addressed the relationship of FABs with disability and behavioral performance in chronic LBP (CLBP) patients. McCracken and colleagues (1993), using a straight leg raise test, as the dependent variable, in a sample of 43 CLBP patients, reported that anxious patients over-predicted pain and terminated the test earlier than non-anxious patients, showing that greater pain anxiety, as measured with Pain Anxiety Symptoms Scale (PASS), is associated with decreased physical performance $(\mathrm{r}=$ -0.36, p < 0.05) [27]. Also, Vlaeyen., et al. (1995), in a correlational study, requested from 31 patients suffering from CLBP to lift and hold a $5.5 \mathrm{~kg}$ weight with their dominant hand until pain made it impossible to continue. Fear of movement re-injury, as measured with the Tampa Scale of Kinesiophobia (TSK), significantly predicted poor behavioral performance $(r=-0,44, p<0.01)$ [18]. Similar findings were reported in a larger sample of 104 patients with CLBP by Crombez., et al. (1999), who as well identified significant correlations $(\mathrm{p}<0.01)$ between a lifting task and a trunk extension flexion unit (Cybex 350 System) and the following fear avoidance measures: TSK ( $r=-0.49)$, FABQ-PA $(r=-0.45)$, FABQ-W $(r=-0.10)$, PASS ( $r=-0.33$ ) [12]. Al-Obaidi., et al. (2000) also confirmed in a sample of CLBP patients $(n=63)$ that FABs, as measured with the Fear avoidance beliefs questionnaire (FABQ), and specifically with FABQ-PA were significant predictors of variation in the spinal isometric strength deficit ( $r=-0.33$ to $-0.43, p<0.01$ ), measured with a Medx lumbar extension machine [28].

Three comprehensive systematic reviews sufficiently addressed the mediating role of fear avoidance in LBP disability development $[25,29,30]$. A systematic review analyzed the intermediate role of fear and other psychological variables (self-efficacy and distress) between pain and disability in 12 studies ( $\mathrm{N}=2,961)$, with 8 of them in LBP, concluded that all those variables were significant mediators. However, the methodological quality of included studies was low [30]. Another systematic review involving both acute and chronic LBP participants (46 independent samples, $N=9,579$ ) reported correlations between pain-related fear and disability of moderate-large magnitude and that these were not affected by demographic or pain characteristics, therefore these relationships develop from an acute stage of symptoms [29]. Furthermore, an additional systematic review including 17 RCTs (5 of high and 12 of moderate methodological quality), additionally examined return to work as an outcome. Findings revealed that high FABs were linked to increased pain and disability levels (in 6 RCTs) and decreased return to work (5 RCTs) and that decreasing FABs during treatment led to less disability and pain at follow-up (2 RCTs), therefore some of the included studies confirmed those relationships [25].

The use of different measures of pain related fear make the direct comparison of these studies difficult. Moreover, the correlational nature of these studies and in many cases the cross-sectional study design does not permit any causal inferences to be made. It is possible that pain related fear may lead to increased activity limitations and resultant disability, but the opposite might be equally true.

\section{FABs in acute LBP}

It has been estimated that more than half of primary care patients with LBP also have elevated fear levels [14]. Therefore, it is of greater importance to tackle the presence of FABs early in the process of back pain. As a consequence, specific interventions in patients who are likely to develop chronic disability might be a viable strategy in order to reduce LBP disability [31]. For this reason, Klenermann., et al. (1995) conducted a prospective study exploring the possibility that FABs are amongst the main determinants of chronicity development in LBP. Biomedical and psychological data were obtained from 300 acute LBP (ALBP) patients within one week and at two months so as to predict the one year outcome. Fear avoidance was among the most powerful predictors of chronic disability 12 months later [32]. However, Vlaeyen and Linton (2000) questioned the use of a non-standardised measure of pain related fear and avoidance used in this study [17]. Fritz and George (2002) examined FABs in 78 ALBP patients. Physical Impairment Index, Oswestry Low Back Pain Disability Questionnaire (OSW), and the Fear-Avoidance Beliefs questionnaire (FABQ) [33], were taken at baseline and 4 weeks later. Elevated FABs were associated with disability and work status, a month later, after controlling for initial levels of pain and physical impairment, proposing that FABQ-W might be the best predictor for return to work for this population [34]. The correlational design and the use of a pain measure not assessed for reliability are limitations of this study. Moreover, the findings cannot be generalized to a non-working population. Also, the same research group in an RCT study $(n=66)$ comparing the 
Addressing Fear Avoidance and Self-efficacy Beliefs to Reduce Disability in Patients with Non-specific Low Back Pain: From Theory to Application

effect of a fear avoidance based physical therapy intervention vs. standard physical therapy, found that patients with ALBP in the experimental group displayed significant improvements in FAB beliefs and self-reported disability relative to the control group, immediately post-intervention (4 weeks) and in the long-term (6 months) [31].

On the other hand, a study investigating the role of FABs in a sample of 615 ALBP patients (workers and non-workers) concluded that FABQ physical activity and work subscales were strongly associated with perceived disability (as measured with the Roland Disability Questionnaire, RDQ) and participation (as measured with an 11-point scale, which included items about participation with home, work, sport, leisure and social activities). Moreover, the measurement of FABs may be valuable in patients with ALBP, regardless of their working status [35]. Among the limitations of this study was the possibility of selection bias, as all referred patients from general practitioners and physiotherapists were not included. Also, all outcome measures were self-reported, representing disability as perceived by the patients rather than actual disability. However, this research group also conducted a study with a dynamic lifting task as an actual performance measure [36]. This task had similarities with the task of 2 previous studies [12,18], but in this study a larger sample size $(n=96)$ of patients with ALBP performed the lifting task. The researchers concluded that pain related fear, as measured with the TSK, was linked to functional status in individuals suffering an ALBP episode.

An additional study which examined FABs in the process of ALBP was that of Sieben., et al. (2002) [1]. Participants completed questionnaires related to back pain history and current episode, Tampa Scale for Kinesiophobia (TSK), Pain Catastrophising Scale (PCS) and Oswestry Disability Questionnaire (ODQ) and for a period of two weeks a diary consisting of several visual analogue scales. In this study, three subgroups of patients were identified: those with descending, with stable and with rising levels of pain related fear. Patients who had rising levels of fear during the first two weeks of a new episode were more disabled than the other two groups. The results of this investigation show that different levels of pain related fear might exist between individuals suffering a new episode of back pain and this probably accounts for the increased disability that some patients report [1]. Nevertheless, all data were based on self-reported measures. Moreover, the sample size was relatively small $(n=44)$ and the methodology for their recruitment was unclear.
Sieben., et al. (2005) in a further study examined the role of FABs in the process of chronicity. In their longitudinal study, questionnaires were completed at baseline, three, six and twelve months follow up and at the end of the study by 174 patients with ALBP. A backward ordinal regression analysis revealed that the most important predictors of future disability were previous LBP history and pain intensity rather than FABs. The authors concluded that the FAM alone may not be sufficient to explain the process by which patients suffering from ALBP acquire chronic disability, therefore there may be additional factors influencing the pathway to chronicity [37]. Nevertheless, fear-avoidant patients were underrepresented in the analyses, thus impacting the statistical power of these results. Moreover, physical activity was examined by an engagement to daily activities rating scale, this being a potential influence to patients' interpretation of the variance of interest.

\section{FABs in different stages of chronicity}

Grotle., et al. (2004) compared FABs in patients with ALBP (less than three weeks, $n=123$ ) and CLBP (more than a year, $n=233$ ). Acute patients were referred to the project for interview and examination before the first contact with the healthcare professional and from advertisement in the local paper. Chronic patients were included after being referred to the department of orthopedic surgery of Oslo's National Hospital. Even though FABs of acute patients were lower than these of chronic LBP patients, in both patient groups FABs were equally strongly associated with disability, making the authors conclude that FABs should be identified at an early stage of LBP [38]. Among the strengths of this study is the use of a large sample, but its nature does not permit to make causal inferences between variables.

Also, Boersma and Linton (2005) compared patients at different stages of chronicity, with 48 patients having LBP less than one year, 47 suffered from pain between 1-3 years and 89 for more than 3 years. Data focused on pain intensity, FABs, catastrophizing and function. However, this study did not find fear of movement to be a significant early predictor of function, not until after 1 year of pain duration [39]. Nevertheless, the particular study design did not allow substantiation of direction of causality among the variables. Furthermore, the participants of this study were recruited by newspaper advertisements so this self-selection process makes the generalisability of the results questionable. Moreover, the stages of chronicity were not divided according to the traditional medical definitions of chronicity, while the patients with pain for less than one year were almost a quarter of the other groups. 
Addressing Fear Avoidance and Self-efficacy Beliefs to Reduce Disability in Patients with Non-specific Low Back Pain: From Theory to Application

FABS - Results from longitudinal monitoring studies in the general population

Longitudinal monitoring studies are useful in order to examine interactions between FABs, pain and disability in the general population. In a study monitoring 36 patients from the general population in a subacute stage of LBP symptoms not seeking medical care while recovering from ALBP, the relationship between pain-related fear and spinal motion was examined. Participants performed a forward bending task at 3,6, and 12 weeks following onset of low back pain. Pain and pain-related fear were correlated with joint excursions observed during each forward bending measurement. Lumbar motion was negatively affected by pain-related fear, but not low back pain, at all three testing sessions. In contrast, hip motion negatively correlated with pain at all three testing sessions but was not related to fear. These findings suggest that painrelated fear results in avoidance behavior that specifically limits or restricts motion of the lumbar spine [40]. However, the sample of this study was rather small.

In a large longitudinal study involving 415 participants with non-spinal pain during the previous years, who filled out a baseline and a follow up questionnaire and performed an 8 self-administered tests, such as rising and squatting, participants with above the median FABs scores at pretest had greater risk of suffering an episode of back pain and almost twice the risk of lower functioning at the one year follow up [41]. Similarly, in another study of 1571 Dutch participants were randomly sampled in order to explore the role of kinesiophobia in LBP, chronic LBP and related disability. In those patients already having LBP at baseline $(n=411)$, a high level of self-reported kinesiophobia, predicted not only the presence of LBP at 6 months follow up and CLBP but especially severe LBP and LBP with disability. Those associations remained significant even after adjustment for pain severity, pain duration or disability at baseline. Additionally, even for individuals without LBP at baseline $(n=1160)$, high levels of fear of pain predicted LBP with disability at the follow up [42]. These findings are important, as they enrich the knowledge regarding the relationship of FABs with chronic disability not only to patients but to pain free individuals as well. In addition, the prospective nature of such studies supports the notion that FABs predict future disability within the same individuals suffering from back pain or not.

Also, in a longitudinal study of a working patient population, the dynamic relationship between pain related fear, pain and disability was examined, using a path analytic approach [43]. Two independent prospective studies were performed, the first in a sample of working employees who experienced LBP at baseline and at follow up (18 months) and the second in young working employees who developed pain at one year follow up. The results of both studies showed that the model where pain related fear is a consequence of pain severity had a better fit than the model in which pain related fear is an antecedent of pain severity. Additionally, when the direct effects were examined, pain severity at baseline proved to significantly explain pain related fear and disability at follow up. The low response rate though of the participants (63\%) in the first study and the lack of measurements of pain severity and pain associated disability at baseline in the second study present some limitations.

\section{The influence of self-efficacy in LBP-related disability}

Research has demonstrated further that there may be other significant cognitive factors influencing the transition to chronic disability in patients with LBP. Findings from studies including both pain related fear and self-efficacy beliefs as predictors of disability report the importance of self-efficacy in explaining disability. Selfefficacy, according to Bandura (1977), is "the confidence the person has in his or her own ability to achieve a desired outcome" [44].

Ayre and Tyson (2001) [45] and Denison., et al. (2004) [46] reported that self-efficacy beliefs are better predictors of disability than pain related fear. The data of these studies though were derived from patients who did not exhibit high pain related fear levels, explaining probably in this way the higher contribution of self-efficacy beliefs.

A study by Foster., et al. (2010) examined 20 psychological factors that may present as obstacles to recovery, 6 months after primary care consultation. The factors that were more predictive of outcome and explained $56.6 \%$ of the variance in disability were the following: firstly, the perception that the problems related to LBP will be long-lasting, secondly that the back problem is the cause of all the symptoms, thirdly, their beliefs that they are unable to take control of their problem and finally their low confidence to their capability of performing normal activities. On the other hand, fear avoidance, catastrophising and depression, in the univariate regression analysis, could not uniquely explain the outcome [47]. Nevertheless, not all possible psychological factors were examined in the study, the 6-month follow up might have been short for this type of research design and there was also selection bias involved, as not all patients who consulted primary care participated in the analysis.

Woby., et al. (2007), upon examination of fear avoidance and self-efficacy beliefs in a sample of 102 CLBP patients who had been referred to an outpatient physiotherapy rehabilitation program and who reported elevated levels of pain related fear, also found self-efficacy to be more strongly associated with pain intensity and disability in comparison to pain related fear [48]. However, Woby., et al. taking into account the findings of the aforementioned study, proposed a modification to the FAM. Specifically, they proposed 
Addressing Fear Avoidance and Self-efficacy Beliefs to Reduce Disability in Patients with Non-specific Low Back Pain: From Theory to Application

that when elevated levels of pain related fear lead to a reduction in functional self-efficacy, then avoidance might occur, which in turns leads to greater disability, depression and disuse. Contrary, avoid- ance is less likely to occur when pain related fear does not contribute to diminished self-efficacy beliefs (Figure 1). A limitation of this study was that it followed a cross-sectional research design.

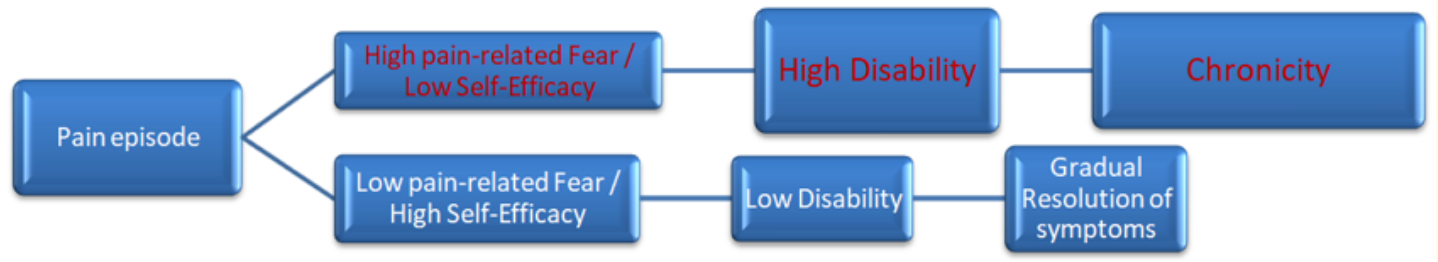

Figure 1: The duration (chronicity) and disability related to a pain episode under the differential combined influence of pain-related fear and self-efficacy.

However, the same research group also conducted a subsequent intervention study in patients with CLBP, analyzing the changes incurred by a physical therapy behavioural modification treatment program. Specifically, the improvements noted in cognitive variables that could explain changes in disability were examined. Findings confirmed that reductions in fear of movement and catastrophizing and increases in functional self-efficacy were all important contributors towards disability improvement (by $17 \%$, after accounting for demographics and pain intensity) in patients with CLBP, even for the 6-week intervention period examined [49]. Therefore, positive interactions between cognitive factors and disability can be brought about rather quickly even in patients with CLBP, however, longer-term follow ups are also required to establish the effectiveness of these interventions in the long run.

Moreover, Costa., et al. (2011) conducted a two wave longitudinal study of 184 chronic LBP patients. The latest completed several measures such as pain intensity, disability, pain self-efficacy and fear of movement. Regression analyses were used in order to test the extent to which fear of movement and self-efficacy mediated the relationship between pain intensity and disability. Measured at the same time, both variables partially mediated the effects of pain on disability at the onset of pain. However, they did not find evidence that fear of movement mediated this relationship over a year period, in contrast to self-efficacy, which partially mediated the relationship between changes in pain and changes in disability [44]. A subsequent large RCT $(n=701)$, indeed, confirmed that improvements in fear of movement and self-efficacy significantly explained disability improvement at 12 months, after a 6-week cognitive-behavioural type of intervention (Back Skills Training )
[50]. The mediation of fear, self-efficacy and distress between pain and disability was also confirmed by a recent systematic review with meta-analysis [30], however the studies included were of low methodological quality.

Clinical physical therapy applications to improve self-efficacy and reduce fear of movement

Physical therapists are among the health professionals that patients come frequently into contact, therefore their clinical reasoning skills either towards a 'biomedical' or a 'biopsychosocial' model of patient care may affect their perceptions of harmfulness of physical activities and suggestions to patients for return-to-work [51]. Therefore, training of physiotherapists within the biopsychosocial assessment and management of musculoskeletal conditions is one of the cornerstones to successful rehabilitation [52].

Different authors have described a similar approach targeting maladaptive cognitions acting as moderators of a successful treatment outcome Based on the transtheoretical model of change [53], effective interventions to enhance the functional abilities of chronic LBP sufferers [54] have been presented, such as the Pain Neuroscience Education (PNE) [55,56] or the Explain Pain [57] approaches. In conjunction, the model of graded activity/exposure to potentially painful stimuli has been extensively validated [58,59]. Details of those interventions are provided in these and other relevant publications.

An initial assessment of coping styles may be necessary in order to handle patients' cognitive impairments more effectively, as participants who have reported high levels of passive coping expe- 
Addressing Fear Avoidance and Self-efficacy Beliefs to Reduce Disability in Patients with Non-specific Low Back Pain: From Theory to Application

rienced a 3-times increase in the risk of persistent disabling low back pain [60]. Therefore, fear avoidance vs. confrontation, as 2 different coping styles of the same construct, need to be thoroughly evaluated prior to intervention. As, Rainville., et al. (2011) suggest, there may be subcategories of avoiders, the 'misinformed avoiders' requiring information to adjust their FABs, the 'learned pain avoiders' requiring pain-desensitizing treatments and the 'affective avoiders' requiring a combination of fear desensitization and counseling [11]. Indeed, successful outcomes have been reported when FABs are incorporated within a structured progressive patient return-to-activity program, either with specific or general trunk activation exercises in combination with challenging their beliefs with written information (The Back Book) [61], or with a more pain-contingent quota-based return to activity [49].

Since the levels of physical activity seem to be inversely related to disability in CLBP [62], a more graded approach seems better suited to patients with CLBP [59]. Since physical activity levels were reported to be variable in acute LBP [62], the speed of return to normal, recreational or even athletic activities needs to be adjusted to patients' physical abilities as well as their FAB levels [63]. The social component of back pain should not be overlooked, and indeed a recent qualitative study has shown that patients with CLBP experience altered social interaction with their family, friends and work colleagues and as a consequence a negative perception of themselves also developed an inability to perform activities of daily living, as part of their social role [64]. Interventions taking into consideration the above therapy principles might be particularly beneficial for patients with either acute or chronic LBP.

\section{Conclusion}

This review examined two of the most important psychological factors which influence LBP-related disability. Fear avoidance and self-efficacy are key variables in the expanded FAM and their contribution on disability has been examined by either cross-sectional or prospective research designs, however, of variable follow up times and different or no interventions. More studies are required to understand this vicious cycle of prolonged and high levels of pain and disability reported by LBP patients in order to diminish the economical, societal and psychological impact of LBP, with appropriate prevention and focused physical therapy interventions delivered under the biopsychosocial framework of patient care.

\section{Conflict of Interest}

The authors declare there is no conflict of any financial or other interest regarding this work.

\section{Bibliography}

1. Sieben JM., et al. "Pain-related fear in acute low back pain: the first two weeks of a new episode". European Journal of Pain 6.3 (2002): 229-237.

2. Waddell, G. "The Back Pain Revolution". 2nd ed. Edinburgh: Churchill Livingstone (2004).

3. Croft PR., et al. "Outcome of low back pain in general practice: a prospective study". BMJ 316.7141 (1998): 1356-1359.

4. Watson PJ., et al. "Medically certified work loss, recurrence and costs of wage compensation for back pain: a follow-up study of the working population of Jersey". British Journal of Rheumatology 37.1 (1998): 82-86.

5. Maniadakis N and A Gray. "The economic burden of back pain in the UK". Pain 84.1 (2000): 95-103.

6. Dagenais S., et al. "A systematic review of low back pain cost of illness studies in the United States and internationally". Spine Journal 8.1 (2008): 8-20.

7. Andronis L., et al. "Cost-Effectiveness of Non-Invasive and Non-Pharmacological Interventions for Low Back Pain: a Systematic Literature Review". Applied Health Economics and Health Policy (2016).

8. Pope MH., et al. "Spine ergonomics". Annual Review of Biomedical Engineering 4 (2002): 49-68.

9. Koumantakis GA. "Muscle activity and back pain". in Topical Issues in Pain 5, L. Gifford, Editor. CNS Press Ltd.: Kestrel, UK (2006): 341-377.

10. Tagliaferri SD., et al. "Domains of Chronic Low Back Pain and Assessing Treatment Effectiveness: A Clinical Perspective". Pain Practice 20.2 (2020): 211-225.

11. Rainville J., et al. "Fear-avoidance beliefs and pain avoidance in low back pain--translating research into clinical practice". Spine Journal 11.9 (2011): 895-903.

12. Crombez G., et al. "Pain-related fear is more disabling than pain itself: evidence on the role of pain-related fear in chronic back pain disability". Pain 80 (1999): 329-339.

13. Asmundson GJ., et al. "Beyond pain: the role of fear and avoidance in chronicity". Clinical Psychology Review 19.1 (1999): 97-119.

14. Bunzli, S., et al. "Making Sense of Low Back Pain and PainRelated Fear". Journal of Orthopaedic and Sports Physical Therapy 47.9 (2017): 628-636.

15. Vlaeyen JW and G Crombez. "Fear of movement/ (re)injury, avoidance and pain disability in chronic low back pain patients". Manual Therapy 4.4 (1999): 187-195. 
16. Swinkels-Meewisse IE., et al. "Fear of movement/ (re)injury predicting chronic disabling low back pain: a prospective inception cohort study". Spine (Phila Pa 1976) 31.6 (2006): 658-664.

17. Vlaeyen JW and SJ Linton. "Fear-avoidance and its consequences in chronic musculoskeletal pain: a state of the art". Pain 85.3 (2000): 317-332.

18. Vlaeyen JW., et al. "Fear of movement/ (re)injury in chronic low back pain and its relation to behavioral performance". Pain 62.3 (1995): 363-372.

19. Lethem J., et al. "Outline of a Fear-Avoidance Model of exaggerated pain perception-I". Behaviour Research and Therapy 21.4 (1983): 401-418.

20. Vlaeyen JW., et al. "The role of fear of movement/ (re)injury in pain disability". Journal of Occupational Rehabilitation 5.4 (1995): 235-252.

21. Melzack R. "Pain and the neuromatrix in the brain". Journal of Dental Education 65.12 (2001): 1378-1382.

22. Melzack R. "From the gate to the neuromatrix". Pain 6 (1999): S121-126.

23. Meier ML., et al. "The impact of pain-related fear on neural pathways of pain modulation in chronic low back pain". Pain Reports 2.3 (2017): e601-e601.

24. Barke A., et al. "Neural Correlates Differ in High and Low FearAvoidant Chronic Low Back Pain Patients When Imagining Back-Straining Movements". Journal of Pain 17.8 (2016): 930943.

25. Wertli MM., et al. "Fear-avoidance beliefs-a moderator of treatment efficacy in patients with low back pain: a systematic review". Spine Journal 14.11 (2014): 2658-2678.

26. Crombez G., et al. "Fear-avoidance model of chronic pain: the next generation". Clinical Journal of Pain 28.6 (2012): 475-483.

27. McCracken LM and RT Gross. "Does anxiety affect coping with chronic pain?”. Clinical Journal of Pain 9.4 (1993): 253-259.

28. Al-Obaidi SM., et al. "The role of anticipation and fear of pain in the persistence of avoidance behavior in patients with chronic low back pain". Spine (Phila Pa 1976) 25.9 (2000): 1126-1131.

29. Zale EL., et al. "The relation between pain-related fear and disability: a meta-analysis". The Journal of Pain: Official Journal of the American Pain Society 14.10 (2013): 1019-1030.

30. Lee H., et al. "How does pain lead to disability? A systematic review and meta-analysis of mediation studies in people with back and neck pain". Pain 156.6 (2015): 988-997.
31. George SZ., et al. "The effect of a fear-avoidance-based physical therapy intervention for patients with acute low back pain: results of a randomized clinical trial". Spine (Phila Pa 1976) 28.23 (2003): 2551-2560.

32. Klenerman L., et al. "The prediction of chronicity in patients with an acute attack of low back pain in a general practice setting'. Spine (Phila Pa 1976) 20.4 (1995): 478-484.

33. Waddell G., et al. "A Fear-Avoidance Beliefs Questionnaire (FABQ) and the role of fear-avoidance beliefs in chronic low back pain and disability". Pain 52.2 (1993): 157-168.

34. Fritz JM and SZ George. "Identifying psychosocial variables in patients with acute work-related low back pain: the importance of fear-avoidance beliefs". Physical Therapy 82.10 (2002): 973-983.

35. Swinkels-Meewisse IE., et al. "Fear-avoidance beliefs, disability, and participation in workers and non-workers with acute low back pain". Clinical Journal of Pain 22.1 (2006): 45-54.

36. Swinkels-Meewisse IE., et al. "Acute low back pain: pain-related fear and pain catastrophizing influence physical performance and perceived disability". Pain 120 (2006): 36-43.

37. Sieben JM., et al. "A longitudinal study on the predictive validity of the fear-avoidance model in low back pain". Pain 117 (2005): 162-170.

38. Grotle M., et al. "Fear-avoidance beliefs and distress in relation to disability in acute and chronic low back pain". Pain 112.4 (2004): 343-352.

39. Boersma K and SJ Linton. "How does persistent pain develop? An analysis of the relationship between psychological variables, pain and function across stages of chronicity". Behaviour Research and Therapy 43.11 (2005): 1495-1507.

40. Thomas JS and CR France. "The relationship between painrelated fear and lumbar flexion during natural recovery from low back pain". European Spine Journal : Official Publication of the European Spine Society, the European Spinal Deformity Society, and the European Section of the Cervical Spine Research Society 17.1 (2008): 97-103.

41. Linton SJ., et al. "Are fear-avoidance beliefs related to the inception of an episode of back pain? A prospective study". Psychology Health 14.6 (2000): 1051-1059.

42. Picavet HS., et al. "Pain catastrophizing and kinesiophobia: predictors of chronic low back pain". American Journal of Epidemiology 156.11 (2002): 1028-1034.

43. Gheldof EL., et al. "Pain-related fear predicts disability, but not pain severity: a path analytic approach of the fear-avoidance model". European Journal of Pain 14.8 (2010): 870.e1-9. 
44. Costa Lda C., et al. "Self-efficacy is more important than fear of movement in mediating the relationship between pain and disability in chronic low back pain". European Journal of Pain 15.2 (2011): 213-219.

45. Ayre M and GA Tyson. "The role of self-efficacy and fearavoidance beliefs in the prediction of disability". Australian Psychologist 36.3 (2001): 250-253.

46. Denison E., et al. "Self-efficacy, fear avoidance, and pain intensity as predictors of disability in subacute and chronic musculoskeletal pain patients in primary health care". Pain 111.3 (2004): 245-252.

47. Foster NE., et al. "Distinctiveness of psychological obstacles to recovery in low back pain patients in primary care". Pain 148.3 (2010): 398-406.

48. Woby SR., et al. "Self-efficacy mediates the relation between pain-related fear and outcome in chronic low back pain patients". European Journal of Pain 11.7 (2007): 711-718.

49. Woby SR., et al. "Outcome following a physiotherapist-led intervention for chronic low back pain: the important role of cognitive processes". Physiotherapy 94.2 (2008): 115-124.

50. Fordham B., et al. "Explaining How Cognitive Behavioral Approaches Work for Low Back Pain: Mediation Analysis of the Back Skills Training Trial". Spine (Phila Pa 1976) 42.17 (2017): E1031-e1039.

51. Houben RM., et al. "Health care providers' orientations towards common low back pain predict perceived harmfulness of physical activities and recommendations regarding return to normal activity". European Journal of Pain 9.2 (2005): 173183.

52. Cowell I., et al. "Perceptions of physiotherapists towards the management of non-specific chronic low back pain from a biopsychosocial perspective: A qualitative study". Musculoskeletal Science and Practice 38 (2018): 113-119.

53. Holden J., et al. "Health coaching for low back pain: a systematic review of the literature". International Journal of Clinical Practice 68.8 (2014): 950-962.

54. Ben-Ami N., et al. "Increasing Recreational Physical Activity in Patients With Chronic Low Back Pain: A Pragmatic Controlled Clinical Trial". Journal of Orthopaedic and Sports Physical Therapy 47.2 (2017): 57-66.

55. Nijs J., et al. "In the spine or in the brain? Recent advances in pain neuroscience applied in the intervention for low back pain". Clinical and Experimental Rheumatology 35.5 (2017): 108-115.
56. Louw A., et al. "The efficacy of pain neuroscience education on musculoskeletal pain: A systematic review of the literature". Physiotherapy Theory and Practice 32.5 (2016): 332-355.

57. Moseley GL and DS Butler. "Fifteen Years of Explaining Pain: The Past, Present, and Future". Journal of Pain 16.9 (2015): 807-813.

58. Woods MP and GJ Asmundson. "Evaluating the efficacy of graded in vivo exposure for the treatment of fear in patients with chronic back pain: a randomized controlled clinical trial". Pain 136.3 (2008): 271-280.

59. López-de-Uralde-Villanueva I., et al. "A Systematic Review and Meta-Analysis on the Effectiveness of Graded Activity and Graded Exposure for Chronic Nonspecific Low Back Pain". Pain Medicine 17.1 (2016): 172-188.

60. Jones GT., et al. "Predicting persistent disabling low back pain in general practice: a prospective cohort study". British Journal of General Practice 56.526 (2006): 334-341.

61. Koumantakis GA., et al. "Trunk muscle stabilization training plus general exercise versus general exercise only: randomized controlled trial of patients with recurrent low back pain". Physical Therapy 85.3 (2005): 209-225.

62. Lin CW., et al. "Relationship between physical activity and disability in low back pain: a systematic review and metaanalysis". Pain 152.3 (2011): 607-613.

63. Wand BM., et al. "Early intervention for the management of acute low back pain: a single-blind randomized controlled trial of biopsychosocial education, manual therapy, and exercise". Spine (Phila Pa 1976) 29.21 (2005): 2350-2356.

64. Bailly F., et al. "The impact of chronic low back pain is partly related to loss of social role: A qualitative study". Joint Bone Spine 82.6 (2015): 437-441.

\section{Assets from publication with us}

- Prompt Acknowledgement after receiving the article

- Thorough Double blinded peer review

- Rapid Publication

- Issue of Publication Certificate

- High visibility of your Published work

Website: https://www.actascientific.com/

Submit Article: https://www.actascientific.com/submission.php Email us: editor@actascientific.com

Contact us: +919182824667 